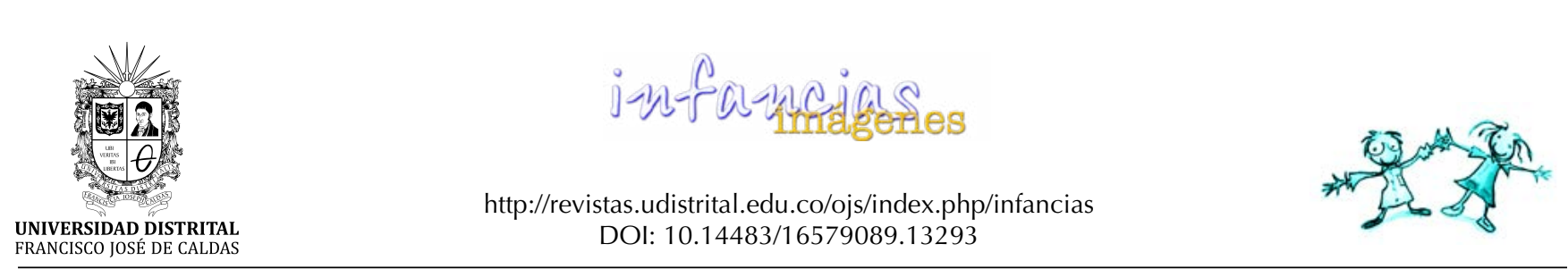

TEXTOS Y CONTEXTOS

\title{
Pensando en la salud de niños y niñas, el aporte desde las representaciones sociales*
}

\author{
Thinking about Children Health, \\ the Contribution from Social Representations
}

\author{
María Fernanda Cañón Rodríguez¹, Daniel Marín², Roberto Fasanelli³
}

Para citar este artículo: Cañón, M. F., Marín, D., Fasanelli, R. (2018). Pensando en la salud de niños y niñas, el aporte desde las representaciones sociales. Infancias Imágenes, $17(2), 197-208$

\section{Resumen}

En este artículo se pretende analizar el concepto de salud infantil. Este ha sido un interrogante permanente y transversal para la sociedad occidental en su esfuerzo por precisar tanto el significado como sus alcances en la vivencia cotidiana, así como en la formulación de las políticas públicas que permitan una acción más acertada de los actores involucrados en su consolidación. Al ser su contraparte la enfermedad, esta figura como una de las principales preocupaciones que requiere ser comprendida y explicada para generar propuestas de solución a los problemas presentados. Los diferentes abordajes reiteran la necesidad de la participación de niños y niñas, reconociendo sus miradas, opiniones, imaginarios, representaciones, categorías emergentes y teorías construidas, las cuales a su vez se relacionan con prácticas y comportamientos cotidianos. Por tanto, ejes transformadores de la reflexión del ejercicio de la salud pública traducidos en la construcción de ciudadanía por la infancia.

Palabras clave: salud materno-infantil, derechos del niño, pediatría, salud pública.
Recibido: 23-marzo-2017 / Aprobado: 18-febrero-2018

\section{Abstract}

This paper aims to analyze the concept of child health. This has been a permanent and transversal question for Western society in its effort to specify both the meaning and its scope in the daily experience, as well as in formulating public policies that allow a more accurate action of the actors involved in its consolidation. Being the disease its counterpart, it appears as one of the main concerns that needs to be understood and explained to generate proposals to solve presented problems. The different approaches reiterate the need for children's participation, recognizing their points of view, opinions, imaginaries, representations, emerging categories and constructed theories, which in turn are related to everyday practices and behaviors. Therefore, transformation axis of reflection of the exercise of public health translates into the construction of citizenship by childhood.

Keywords: maternal-child health, children rights, pediatrics, public health.

\footnotetext{
* Artículo de revisión. Ensayo que hace parte de investigación "Representaciones sociales de salud infantil en niños, niñas y equipo de atención en salud, en contextos rural y urbano del departamento de Cundinamarca", para optar al grado de candidatura a doctor en Salud Pública. Financiado por Colciencias, convocatoria nacional n. ${ }^{\circ}$ 617. Iniciada en febrero de 2015 y actualmente en curso.

Médica Pediatra, Especialista en pedagogía, Magíster en Desarrollo Educativo y Social, PhD (c) Doctorado en Salud Pública, Universidad Nacional de Colombia. Correo electrónico: mfcanonr@unal.edu.co

Psicólogo. Magíster en Desarrollo Educativo y Social, Universidad de Manizales-Cinde. Correo electrónico: danielumanizales@gmail.com Profesor, Departamento de Ciencias Sociales, Università degli Studi di Napoli Federico II. Correo electrónico: fasanell@unina.it
} 


\section{Introducción}

En la infancia nos construimos como seres humanos, individuales y sociales. Además, esta es considerada como definitiva para el futuro de los países. La investigación ha demostrado que "los efectos adversos del pobre desarrollo en la primera infancia tienen efectos negativos a largo plazo en salud, productividad y bienestar general cuando los niños alcancen la adultez" (World Bank Group, 2017, p. 78). Por ello, niños, niñas y adolescentes deben ser prioridad en los escenarios económicos y políticos de nuestro tiempo, pues se considera una población de interés especial en la dinámica de la agenda pública y prioridad en las políticas que afecten sus condiciones de bienestar y salud.

Sin embargo, la realidad de los niños y las niñas es otra en virtud de su vulnerabilidad. Considerando los índices significativos de enfermedades por causas prevenibles, estos requieren consideraciones particulares e intervenciones sociales, económicas, educativas y en atención clínica que se traduzcan en mejoría de indicadores trazadores. Lo anterior requiere de una reflexión de todos los sectores, así como una concepción de salud propia y diferencial que relacione las necesidades y las condiciones del contexto. Ante la dificultad de una definición holística que involucre lo integral del ser, y en la que converjan las diferentes dialécticas y traducciones de esta, se hace necesario develar la construcción social del concepto desde la mirada de los actores que intervienen en ella para comparar entre lo cotidiano y lo establecido y así poder vislumbrar si este es coherente con los enfoques teóricos y las acciones institucionales propuestas, tanto en lo rural como lo urbano.

\section{Infancia y salud}

Históricamente el término salud ha presentado transformaciones en su significado, de acuerdo con las condiciones de cada época e interpretaciones a la luz del conocimiento acumulado, orientando el ejercicio de la salud pública y la atención individual. De igual modo, es fruto de la negociación entre el saber científico y el saber común socialmente compartido y construido. Por tanto, implica también un análisis del concepto de salud y una deconstrucción de este para generar discursos coherentes con la realidad y con la situación de salud y enfermedad de los niños. Es decir, una construcción integral y colectiva que involucre su pensamiento y la representación social fundada desde su propia vivencia. Como lo plantea Rosemberg "saldar la deuda con la infancia es traer al niño o la niña, su familia y sus necesidades al espacio público, fortaleciendo su poder de negociación en la arena pública del debate sobre las prioridades en políticas públicas" (2009, p. 24).

El poder comparar y establecer relaciones entre estos saberes hacia la comprensión de las construcciones sociales de la salud como proceso, comprendiendo su dinámica, permitiría no solo acercar las políticas públicas a los niños y niñas, sino también hacerla parte de su elaboración. Como lo afirma Contandriopoulos:

El sentido que los diferentes actores afectados dan a la vida, a la muerte, al dolor, a la enfermedad y el nivel de análisis considerado constituye el sistema simbólico que permite pensar la salud en sus diferentes dimensiones y por ende actuar sobre ella [...]. Las representaciones de la salud y de la enfermedad dependen de la posición ocupada en la sociedad y en particular de la relación que una persona tiene con el campo médico. (2006, p. 7)

Comprender el binomio salud-enfermedad por parte de niños y niñas da elementos para la articulación entre la gestión pública, el compromiso de la sociedad y las condiciones de vida de la infancia, lo cual requiere que los intereses y problemáticas vividas por ellos sean elementos importantes en el agendamiento de sus prioridades, así como su posicionamiento público y gubernamental. De esta forma, se legitiman los procesos de formulación y elaboración de políticas, fortaleciendo a los agentes de cambio para la transformación de su realidad social. Por otra parte, la importancia de la reflexión en los programas de formación de profesionales de la infancia crea un desafío para la comprensión de la relevancia de los factores culturales en las respuestas de las poblaciones a la prevención, tratamiento y manejo de condiciones que amenazan la vida, así como en las prácticas ligadas a la promoción del bienestar infantil. 


\section{¿Cómo se ha comprendido la salud infantill?}

Los problemas conexos con la salud infantil se han caracterizado y analizado ampliamente en la literatura especializada. Es así como en el siglo XVIII se plantea la relación entre procesos de industrialización y de urbanización con las inadecuadas condiciones de cuidado, alimentación y seguridad para niños y niñas pertenecientes a la clase trabajadora (Rosen, 1993). Hacia la mitad del siglo XIX, Engels (citado por Starfield 2008) documentó que las tasas de mortalidad de viruela, sarampión, fiebre escarlatina y tos ferina eran mayores entre los hijos de familias de clase trabajadora comparados con los de clase alta; identificó a su vez las difíciles condiciones de la infancia relacionadas con las circunstancias sociales y sentó las bases para el estudio de los mecanismos por los cuales las desventajas sociales producen peor salud, aspecto reiterado por Virchow en su informe epidemiológico (Waitzkin, 2006). Camino al cual se sumaron los aportes de la bacteriología y de la ciencia al finalizar el siglo XIX que permitieron la comprensión de la historia natural de las enfermedades infecciosas, con avances importantes como el desarrollo de vacunas, lo que permitió su control con alto impacto en la mortalidad y morbilidad de la población infantil.

El siglo XX estuvo enmarcado por dos guerras mundiales, el crecimiento de la industria, la congestión de las comunidades urbanas, la profundización de problemas sociales como pobreza, malnutrición, enfermedad e inequidad. Esta situación requirió la formulación de programas con acciones preventivas, de detección de factores de riesgo y la creación de entidades dedicadas a solucionar problemas de la infancia, las cuales promovieron la relación entre el cuidado y la idea de bienestar. A partir de estos, se ha logrado disminuir indicadores como tasa de mortalidad infantil y la tasa de mortalidad en menores de cinco años, datos que muestran la supervivencia de niños y niñas. Durante el siglo XX se resalta los cambios en la estructura y composición de la familia, traducidos en la organización social, y el ingreso de la mujer al mundo laboral, hechos que transformaron las condiciones cotidianas de vida (García-Caballero, 2000b). Un hito fue la firma de la Convención
Internacional de los Derechos del Niño (CIDN) en 1989 y la posterior adopción por los estados que hacen parte, en la cual se hace explícita la garantía en la máxima medida posible del derecho a la supervivencia, lo que derivó directamente en el desarrollo y en la atención en momentos cruciales de la enfermedad.

Este rápido recorrido histórico permite también identificar tendencias teóricas del concepto de salud infantil, las cuales pueden articularse con los modelos en salud pública propuestos por Hernández-Girón, Orozco-Núñez, E. y Arredondo-López (2012), enfoques identificados y adaptados como se describen a continuación.

Enfoque Biomédico: al indagar por la definición de "salud infantil" en la literatura científica, el eje principal de estudio es el biológico, aportando a los importantes avances científicos y tecnológicos que han modificado drásticamente la supervivencia de la población. No obstante, esta producción científica se orienta principalmente hacia la enfermedad tal y como lo había planteado Sigerist (1974, citado por Gómez, 2002), "la noción de enfermedad ha sido central en el discurso médico de la sociedad occidental positivista, [...] desde cuyo nicho comienza a ejercer en casi todo el mundo, una marcada influencia sobre los demás componentes de la utopía; en especial sobre la salud pública y la gestión de políticas públicas en salud" (p. 3). Este enfoque concibe la salud infantil como ausencia de enfermedad y de disfuncionalidad, cuyo propósito es explicarlas y solucionarlas con tratamientos específicos. Apoyándose en investigación epidemiológica, basada en hallazgos clínicos médicos y de laboratorio con avances en métodos diagnósticos, tratamiento y rehabilitación, como son las vacunas y la terapia antibiótica (García-Caballero, 2000a).

- Enfoque epidemiológico: el elemento central de análisis es la identificación de factores de riesgo (Arredondo, 1992). Por tanto, el concepto de prevención de la enfermedad cobra especial relevancia ante la posibilidad de intervenir en mortalidad y morbilidad evitable en patologías infecciosas o crónicas relacionadas con modificaciones de hábitos de vida. Se considera la salud como ausencia de enfermedad y se vincula 
la importancia de elementos como el ambiente y los comportamientos de las personas.

- Enfoque socio histórico: enmarcado en la salud colectiva al reconocer el "carácter social del proceso salud enfermedad en cuanto a fenómeno material objetivo y tal como se expresa en el perfil patológico de los grupos humanos" (Laurell, 1994, p. 6). Epistemológicamente, se ubica en la teoría crítica con implicaciones metodológicas desde la epidemiología social y determinación social. Como lo muestran Carmona y Correa en su investigación (2014), en la cual analizan la relación entre desnutrición, desarrollo infantil y condiciones de vida, muestran que un tercio de los niños estudiados entre tres y cuatro años tienen desnutrición crónica y alguna limitación en la medición del desarrollo infantil, cuya situación de fondo se relaciona con los escasos ingresos económicos, inestabilidad laboral y bajo nivel educativo de la familia; lo que se traduce en necesidades básicas insatisfechas. La relación salud-enfermedad se evidencia como proceso inacabado en el que se involucra necesariamente lo social (Quevedo-Vélez, 2007).

- Enfoque de promoción de la salud: se hace prioritario identificar los factores determinantes sobre los cuales las intervenciones deberían enfocarse, con el interés de asegurar la funcionalidad y el envejecimiento saludable ligados al concepto de bienestar. La salud infantil "está influenciada por múltiples factores determinantes, sociales, ambientales, económicos y genéticos", definición articulada con lo planteado por la Organización Mundial de la Salud (1946, p. 14) y el enfoque de intervención planteado desde la Carta de Ottawa (1986), los cuales vinculan disciplinas de las ciencias de la salud y de las ciencias sociales; en estas se consideran las etiologías como procesos multifactoriales que requieren abordajes interdisciplinarios con una mirada longitudinal y poblacional. Este enfoque da luces a definiciones como la planteada por Wizemann y Anderson (2009), quienes consideran que la salud infantil "involucra algo más que proporcionar acceso a la atención médica. Salud esta integralmente relacionada con el desarrollo y al aprendizaje, y lo que sucede en la vida temprana tiene consecuencias en toda la duración de la vida" (p. 14).

Sumado a los anteriores, se plantea el enfoque económico, en el cual la salud infantil se considera un determinante de las condiciones financieras de los países. Este enfoque la prioriza como proyecto de inversión con una explícita visión en términos de productividad, pues al invertir en la infancia se favorece el capital humano y económico (Irwin, Siddiqi y Hertzman, 2007). Como lo describe el Banco Mundial "las intervenciones que mejoren la nutrición, el desarrollo cognitivo y socioemocional en etapas iniciales de la vida, puede lograr retornos en las etapas posteriores que superan con creces sus costos" (Alderman, Behrman, Glewwe, Fernald y Walker, 2017, p. 94). El fomento de la salud durante los primeros años de vida es relevante para lograr el óptimo desarrollo infantil, por tanto "invertir en la niñez también es importante desde el punto de vista práctico, pues redunda en beneficio de las economías y| las sociedades" (Unicef, 2018).

Este último enfoque tiene relación con el concepto de capacidades, competencias y funcionalidad, identificadas en la definición de salud infantil propuesta por Felix et al. (2014):

Estado dinámico, no simplemente ausencia de enfermedad o de discapacidad, pero también adecuada resiliencia que permita un funcionamiento óptimo físico, mental y social y una óptima calidad de vida en pro de lograr potencial total y el inicio de la independencia funcional y social individual. (p. 49)

En los enfoques descritos y las apuestas ontológicas que subyacen a las definiciones se observa que siguen una línea temporal, relacionada en principio con la enfermedad y con la capacidad productiva posteriormente. Así mismo, es clara la preferencia conceptual de niño como objeto de intervención, en contraposición a la consideración como sujeto de derechos, evidenciando la nula participación de niños y niñas que trasciende el mero ejercicio académico y se retoma en las prácticas de una "vida saludable".

Sin embargo, al realizar un ejercicio dialéctico al interior de la sociedad, la salud infantil atraviesa 
evidentemente el contexto, la memoria colectiva y aspectos culturales, en diálogo permanente con la ciencia y sus diferentes posturas para reconocer las limitaciones de los enfoques propuestos y la puerta a propuestas de concepciones ontológicas que involucren todos estos aspectos. Ejemplo de lo anterior es el indagar sobre las representaciones sociales (RS) de salud infantil, visualizando el camino para develar las necesidades propias de las familias, especialmente los niños y las niñas, además de observar la transformación social y profesional que se ha dado a partir del desarrollo del cada vez más amplio conocimiento científico y técnico.

\section{Representaciones sociales de la salud infantil}

Serge Moscovici definió las RS como un sistema de valores, ideas y prácticas que permiten establecer un orden social y facilitar la comunicación y transformación de lo no familiar en familiar, condición necesaria para construir el mundo social. Es así como la teoría de las representaciones sociales (TRS) estudia el conocimiento del sentido común como una construcción útil en la cotidianidad, que proviene de la producción de toda una serie de informaciones que se transforman e integran antes de reapropiarse. En palabras del autor, se estudia el proceso de "cómo las personas transforman una pieza de conocimiento, crean información que luego confirman, y simultáneamente objetivan en sus prácticas diarias" (Moscovici, 1988).

Una de las primeras autoras que aborda la comprensión de las RS de la salud y la enfermedad es Claudine Herzlich, quien en 1969 refiere que "estudiar la representación social de la salud y la enfermedad, es observar como el conjunto de valores, de normas sociales y modelos culturales se piensan y viven por los individuos de nuestra sociedad" ( $p$. 13), siendo el binomio salud-enfermedad relevante en el establecimiento y actualización de relaciones y ajustes del individuo o la sociedad, postura que trasciende el enfoque desde el sistema sanitario. En su obra, la autora identifica tres corrientes psicosociales sobre la enfermedad y la práctica médica, como son la medicina psicosomática, la relatividad cultural y la importancia de los comportamientos sociales concernientes a salud y enfermedad (Banchs, 2007).
Bajo una concepción pluralista de la salud, refiere que no es posible hablar de "la salud", sino de "las saludes", identificando en su investigación tres formas: salud vacía, fondo de la salud y equilibrio. La primera se relaciona con la ausencia de enfermedad, la segunda como un capital dinámico - sea de vitalidad o de resistencia a la enfermedad-considerando que la infancia es un periodo particularmente propicio para desarrollar un buen fondo de salud, y la tercera como bienestar definido como un "todo o nada" (Herzlich, 1969, p. 79). De modo que cada grupo social construye su propia representación, rescatando el peso de lo cultural en la elaboración del saber socialmente compartido; una relación dinámica en constante cambio y cuyas representaciones se influencian interdependientemente con las prácticas cotidianas. De acuerdo con Jodelet (2013) "el significado de la salud y de la enfermedad debe entenderse en el contexto cultural e histórico que a nivel social y colectivo induce representaciones y sus valores, estos determinaran el significado dado por los individuos que atraviesan las experiencias" (p. 13), lo que permite concebir que salud-enfermedad es una construcción social en la cual convergen no solo los presupuestos académicos consensuados científicamente, sino también el constructo de los grupos sociales, sea desde su contenido oral tradicional o desde la apropiación de saber por diferentes manifestaciones del lenguaje, además de los símbolos, significados y significantes que concentran su propia RS y que sirven de base inicial para la explicación y vivencia de las misma. Es decir, todas las dinámicas internas y externas entran en juego con los conocimientos adquiridos, recreados y subsumidos en el discurso que acompaña los signos de enfermedad o la presentación de los síntomas y sus posibles diagnósticos y soluciones. Lo anterior es expresado en el diálogo entre Serge Moscovici e Ivana Markova "cuando se habla de un sistema de conocimiento científico, también se puede hablar de un sistema de conocimiento de sentido común, pero que también está relativamente bien estructurado y es muy rico" (Markova, 1990).

La complejidad en la construcción del concepto de salud para niños y niñas se puede observar en investigaciones como las de Altman y Revenson 
(1985), Campbell (1975), Myant y Williams (2005), Pidgeon (1985) y Piko y Bak (2006), quienes trabajaron con niños entre los 4 y 14 años, y aunque su objetivo específico no es la identificación de las RS, sí tienen en común el propósito de identificar los cambios tanto de conceptos estructurados, como de percepciones sobre la salud y la enfermedad en relación con la edad y el grado de desarrollo de los niños. En general, los autores concuerdan sobre la relación entre la concepción de salud-enfermedad, la edad cronológica y sus experiencias previas. Es decir, identifican en los niños más pequeños la tendencia a definir salud-enfermedad desde el modelo biomédico, mientras que a mayor edad hay mayor complejidad de sus respuestas, con visiones más holísticas y mayor cantidad de elementos en su estructura integradas claramente con las vivencias de cada uno de ellos. Estas son conclusiones similares al trabajo realizado por María del Carmen Vergara (2009), quien busca comprender las RS sobre salud en jóvenes de Manizales, Colombia, y cuyos resultados muestran la salud como un equilibrio mental, material, espiritual, emocional y moral muy vinculado al contexto de violencia, derechos humanos y paz.

Galli y Fasanelli (1995) examinaron las representaciones de salud y enfermedad en niños y niñas de colegios de la ciudad de Nápoles (Italia). Los autores concluyen que los niños no piensan en abstracciones, sino que piensan en realidades; es así como salud se representa desde la acción, el movimiento, la alimentación, la felicidad y la enfermedad desde la inactividad, la terapia y las experiencias personales. Otros estudios corroboran dichos hallazgos, en los cuales niños y niñas relacionan la salud a la adherencia a prácticas saludables, sentimientos positivos y funcionabilidad; mientras que la enfermedad se representa desde los problemas o síntomas específicos, compromiso de la funcionalidad y sentimientos negativos (Cromack, Bursztyn y Rangel, 2009; Lima y de Lemos, 2014).

Identificar dichas RS es una condición para que la sociedad promueva la salud. Solamente por este camino se espera realizar programas efectivos de prevención de la enfermedad y promoción de la salud (Galli y Fasanelli, 1995), pues permiten explicar cómo los miembros de un grupo conceptualizan y se posicionan con relación a una enfermedad, un paciente y un comportamiento preventivo, lo que requiere del enfoque multidisciplinario para su comprensión (Galand y Salès-Wuillemin, 2009). Por otra parte, son importantes al determinar los comportamientos en salud (Knapp, León, Mesa y Suárez, 2003), considerando que este comportamiento se podrá controlar para el mantenimiento de la salud (Бовина et al., 2018). De lo anterior es posible concluir que la mayoría de las investigaciones de RS sobre salud se elaboran para fortalecer y mejorar programas de educación para la salud, en los que con pocas excepciones se reconoce el papel activo de los niños y niñas en la construcción de ideas (Jiménez-Balam, 2012). Incluso, como derivado de estos estudios, se resalta la necesidad de trabajar con temas como la representación de la muerte, pues se evidencia que hay dificultad por parte de los adultos a la hora de trabajar este tema, el cual consideran necesario abordarlo ante la aproximación infantil a la actitud negativa adquirida por el adulto (Žaloudíková, 2010).

No parece común del pensamiento de querer contextualizar las necesidades de niños y niñas y, más aún, la RS que tienen de salud-enfermedad. Osses et al. (2014) muestran la escasa producción teórica sobre la comprensión de la salud de forma integral y plantean la necesidad de continuar la investigación sobre el tema. Se evidencia, entonces, la divergencia entre considerar constitucionalmente a los niños como sujetos de derecho y el ser beneficiarios de atenciones que buscan el control de patologías o el control del comportamiento.

Es más frecuente identificar estudios sobre RS que involucren otros actores como el equipo de atención de salud y los padres. Eronen, Pincombe y Calabretto (2010) muestran el papel de enfermería en el soporte a los padres, los cuales se empoderaron en decisiones acerca del cuidado infantil y devela la necesidad de construcción de la capacidad de los padres para tomar sus propias decisiones acerca del cuidado de niños y niñas. En Latinoamérica se encuentran trabajos en padres y cuidadores, como el elaborado por López-Huerta, Álvarez-Bermúdez y González-Romo (2012) sobre la experiencia psicosocial de padres de hijos con leucemia en un hospital público; o el de González-Martínez, Hernández-Saravia y Correa-Mulett 
(2013) sobre la higiene bucal en madres y cuidadores de hogares infantiles, en los que se destacan aquellas concepciones de temor e incertidumbre por parte de los padres en los problemas que presentan sus hijos. Estos hallazgos permiten proponer a los investigadores acciones dirigidas al apoyo de la población y evidencian factores que involucran el éxito o fracaso en ciertas intervenciones diseñadas para promover, prevenir o tratar. En Argentina, Corvalán (2013a) publicó una investigación en la cual analiza las RS sobre salud-enfermedad infantil en cuidadores, docentes y profesionales de la salud en dos espacios sociales con NBI. Los resultados indican que las RS de la salud en madres son lineales a las prácticas sanitarias centradas en un modelo biomédico, del mismo modo, los maestros pocas veces se reconocen como protagonistas del proceso de salud del niño, mientras que los profesionales de la salud (neurólogos o pediatras) las relacionan con el equilibrio biopsicosocial que corresponde al niño sano y que en ocasiones se contradice con la práctica centrada en la concepción biomédica.

Así, definir salud infantil ha sido un interrogante permanente y transversal para la sociedad Occidental en el esfuerzo por precisar tanto el significado como sus alcances en la vivencia cotidiana y en la formulación de las políticas públicas que beneficien a la población blanco y a los actores que desde las disciplinas de la salud (y otras alternas a estas) intentan mejorar las condiciones de vida saludable de los niños, niñas y adolescentes en todos los escenarios posibles y espacios geográficos locales, nacionales y globales. Existen marcos conceptuales que permiten ubicar la evolución del conocimiento acumulado; sin embargo, "solo un enfoque multidimensional podría dar cuenta de la complejidad de los procesos relacionados con la salud y la enfermedad, así como la diversidad de la experiencia de las personas y su participación en la atención" (Jodelet, 2013, p. 14)

Este esfuerzo implica comprender que la medicina y la salud son campos en los que convergen la construcción ante todo social y que incluyen elementos del contexto simbólico y representacional en la vivencia de las enfermedades. Como lo describe Ofman (2012) "las enfermedades pueden ser entendidas de diversas formas, según el punto de vista del actor social a que se haga referencia", lo que trasciende la atención tradicional y exclusiva de lo mórbido y considera la salud como fenómeno positivo que parte del saber para comprender, el hacer e identificar el qué hacer, así contribuir en la vida de niños, niñas, sus familias y, por ende, de la sociedad.

Esta referencia da un paso a la construcción del mismo concepto, involucra las miradas holísticas de los profesionales de la salud en la que se integren no solo los elementos técnicos del aprendizaje académico, sino también aquellos signos ligados a la salud y la enfermedad, entendiendo esta última como un conjunto de situaciones que hacen que la cotidianidad de los niños discurra desde una normalidad latente a una alteración de la misma a través de los síntomas. Estos últimos son signos en los que posiblemente se evidencia una relación entre las condiciones sociales, psicológicas, nutricionales, culturales y de convivencia familiar que afectan directamente el bienestar, no solo del sujeto sino de su familia y de su entorno.

La lectura, entonces, debe transitar desde las lógicas construidas en el entendimiento de lo técnico del profesional de salud a los entornos macro y micro que implican una reconstrucción de esa lógica y el empoderamiento de una salud y enfermedad desde la vivencia del actor primario, sus relaciones históricas y representaciones, además de sus círculos afectivos y sociales. Siendo coherentes con la intrincada dinámica social, es "indispensable conjugar estas prácticas de salud-educación con los distintos saberes: disciplinas científicas y conocimientos populares, superando así los cercos reduccionistas" (Corvalán, 2013b), enfoques que desafortunadamente Ilevan a la generalización de intervenciones, perdiendo el protagonismo de cada una de las personas como gestoras de su propio proceso, lo que fomenta la dependencia de las comunidades a la intervención dirigida.

La enfermedad en estos términos amplia el rol de los profesionales de salud cuando hace un llamado a descentrar su acción clínica para entrar en una relación de la RS desde la mirada ideológica y experiencial de los niños. Es decir, actuar en los escenarios en los que se encuentran, dando pie a comprender que la salud dista mucho del ambiente hospitalario, pues es dinámica, relativa a las 
vivencias culturales y a las creencias sociales que imprimen un sello único a la experiencia tanto de la salud como de la enfermedad. Esto sin desconocer que a su vez los profesionales de la salud apropian desde su razonamiento reconstruido una apertura, un rompimiento de su mirada clínica, la cual es una mirada que sin dejar de tener esta connotación permita integrar en su diagnóstico y acción curativa las lógicas propias de las comunidades, sus representaciones y creencias. Pero, a su vez, invitando a todos aquellos que tengan que ver con niños y niñas, es decir, a la sociedad en general, a hacerse participes de esta diada.

Contrario a lo anterior, y como lo describe Morin (2006), "el espacio profano de la salud está estructurado hoy por el doble atractivo del riesgo a controlar y el más saludable de conquistar", al concebir la salud predominantemente como ausencia de enfermedad o como un "completo estado de bienestar físico, emocional y social" (Organización Mundial de la Salud, 1946), pone en el centro al individuo, desconociendo aspectos culturales relevantes y la relación directa e indirecta que existe entre este y la sociedad. En estas circunstancias el individuo responde como símbolo a los estándares establecidos en los entornos institucionales pero es leído como unidad útil de las estadísticas, y no desde las miradas humanistas que lo perciben como un ser complejo, posmoderno y que se encuentra inmerso en un contexto en el que se relaciona no solo con sus demás pares sino con su ambiente en una vivencia sistémica que garantiza su supervivencia en cuanto que sus acciones se ven afectadas por el entorno; el cual él también influencia, siempre y cuando esté en constante movimiento.

Así mismo, esto presupone que la salud implica un bienestar determinado por las relaciones ambientales y culturales, condición que amerita entender que la ausencia de enfermedad no existe, lo que existe es un ruido constante que se complejiza y se hace caótico en la medida que los actores primarios son vulnerables a los cambios en toda la dinámica de su esfera de vida. La relación entre infancia y salud pública implica, además de la identificación como población vulnerable e intervenible para disminuir la mortalidad y morbilidad evitable, el considerarla relevante para involucrar en esta etapa vital acciones de prevención de la enfermedad y de tratamiento específico, esperando reducir la carga de enfermedad por patologías infecciosas y crónicas que puedan presentarse en edades posteriores de la vida. Sin embargo, su tarea no para allí, al considerar la salud como derecho fundamental y su principio de prevalencia, la salud pública se reafirma como un campo de acción donde confluyen disciplinas que tienen como propósito hacer efectivo ese derecho y, como lo plantea Franco (2006), "el objeto de estudio es en esencia las interacciones sociales y campos de poder", incluyendo a todos los actores responsables en su materialización.

Por tanto, la salud infantil se define en este documento como un proceso social, históricamente construido y adaptado a los contextos de cada sociedad, los cuales se encuentran en modificación permanente. De esta forma, la salud es una relación dinámica que implica esas relaciones ambientales que surgen desde el mismo momento de la fecundación y que acompañan al ser durante toda su vida, razón de más para entender que va a depender de la forma como vivencie estas relaciones, la buena o mala salud y la conceptualización que se tiene de la misma.

El problema central es que de cierta forma se armoniza un ser complejo, que se ubica en la posmodernidad, pero que es leído en sus enclaves de bienestar y salud desde una mirada moderna (es decir, clásica), con estándares que no responden a los llamados de la sociedad actual. Lo anterior, para intentar hacer un llamado a una nueva mirada de lo que significa salud en Colombia, más que un bien, es un derecho en el que todos somos responsables y con el cual es un deber abogar en las necesidades humanas nacidas del ejercicio social y no exclusiva de los espacios institucionales que no convergen a las realidades respecto de la salud en escenarios nacientes.

\section{Conclusiones}

La relevancia dada a la salud de los niños y las niñas se ha construido históricamente en estrecha relación con las concepciones de infancia y de la salud misma; pasando desde la indiferencia a la supervivencia y posterior consideración como recurso fundamental de la sociedad. Es claro que en cada uno de los diferentes abordajes se encuentra 
ausente la participación de niños y niñas, invisibilizándolos y perpetuando la concepción de objeto de intervención, lo que termina por desconocerlos como sujetos de derecho.

Solo a partir de enfoques que permitan reconocerlos como actores activos en su comunidad, sin perder de vista su singularidad, se lograrán intervenciones acertadas y coherentes que orienten las prácticas cotidianas que tendrán relevancia en su vida. Concepciones que pueden partir del movimiento o la felicidad, hacia la construcción de conceptos contextualizados, los cuales pueden derivar en la transformación de la concepción de salud desde su propia mirada y, por tanto, reconocer a niños y niñas como transformadores de sí mismos, sus familias y sus comunidades.

Todo esto conlleva a repensar la salud como un escenario en el cual su acción debe ser ejercida desde los niños y las niñas. Es allí donde se establecen los primeros eslabones entre bienestar y salud como diadas y vivencias de identificación y de construcción simbólica, razón que implica la estructuración de un sistema de salud integral que permita el acercamiento del niño a espacios de intervención y prevención en los que él mismo participe como actor principal que recree en sus momentos de juego, alegría, alimentación, afecto y demás. Un bienestar integral que harán en el presente ciudadanos comprometidos con su propia vida y la de los demás, sin idealizar el esperado equilibrio entre esferas mentales, afectiva y social. En otras palabras, haciendo real la vinculación del derecho a la salud con el desarrollo integral del ser humano.

\section{Referencias}

Alderman, H., Behrman, J., Glewwe, P., Fernald, L. y Walker, S. (2017). Evidence of Impact of Interventions on Growth and Development during Early and Middle Childhood. En D. Bundy, N. de Silva, S. Horton, D. Jamison y G. Patton (eds.), Disease Control Priorities: Child and Adolescent Health and Development (vol. VIII, pp. 79-98). Washington: International Food Policy Research Institute. Recuperado de http:// dcp-3.org/sites/default/files/volume_downloads/DCP3\%20CAHD_Front\%20Matter.pdf
Altman, D. G. y Revenson, T. (1985). Children's understanding of health and illness concepts: a preventive health perspective. The Journal of Primary Prevention, 6(1), 53-67. DOI: https:// doi.org/10.1007/BF01325340

Arredondo, A. (1992). Análisis y reflexión sobre modelos teóricos del proceso salud-enfermedad. Cadernos de Saúde Pública, 8(3), 254-261. Recuperado de www.scielo.br/pdf/csp/v8n3/ v8n3a05.pdf DOI: https://doi.org/10.1590/ S0102-311X1992000300005

Banchs, M. A. (2007). Representaciones sociales y salud. En T. Rodríguez Salazar y M. L. García Curiel (eds.), Representaciones sociales. Teoría e investigación (pp. 219-254). Guadalajara, México: Universidad de Guadalajara.

Campbell, J. D. (1975). Illness is a point of view: the development of children's concepts of illness. Child Development, 46(1), 92-100. DOI: https://doi.org/10.2307/1128837

Carmona, J. y Correa, A. (2014). Determinación social de la desnutrición y el retardo sicomotor en preescolares de Urabá (Colombia). Un análisis con la epidemiología critica. Facultad Nacional de Salud Pública, 32(1), 40-51. Recuperado de http://www.scielo.org.co/scielo. php?pid=S0120-386X2014000100005\&scrip$\mathrm{t}=$ sci_abstract

Contandriopoulos, A. P. (2006). Elementos para una "topografía" de concepto de la salud. Rupturas. Revista Interdisciplinaria de la Salud, 11(1), 86-99. Recuperado de http://studylib.es/ doc/992017/contandriopoulos--andr--pierre.-2006----elementos-para-u

Corvalán, F. (2013a). Repensando las prácticas de salud y educación en las complejidades sociales. Descripción y análisis de una metodología de investigación acción participativa. Psicogente, 16(26), 197-208. Recuperado de http:// portal.unisimonbolivar.edu.co:82/rdigital/psicogente/index.php/psicogente

Corvalán, F. (2013b). 50 años de representaciones sociales y psicología: Campo psy, bifurcaciones y desafíos. Ecos, 3(1), 115-127. Recuperado de www.periodicoshumanas.uff.br/ecos/article/ viewFile/1055/823 
Cromack, L., Bursztyn, I. y Rangel, L. (2009). O olhar do adolescente sobre saúde: um estudo de representações sociais. Ciência \& Saúde Coletiva, 14(2), 627-634. Recuperado de https://www.scielosp.org/pdf/csc/2009. v14n2/627-634 DOI: https://doi.org/10.1590/ S1413-81232009000200031

Eronen, R., Pincombe, J. y Calabretto, H. (2010). The role of child health nurses in supporting parents of young infants. Collegian, 17(3), 131-141. DOI: https://doi.org/10.1016/j. colegn.2010.04.001

Felix, J. F., Voortman, T., van den Hooven, E. H., Sajjad, A., Leemakers, E. T., Tharner, A. y Franco, O. H. (2014). Health in children: A conceptual framework for use in healthy ageing research. Maturitas, 77(1), 47-51. DOI: https://doi.org/10.1016/j.maturitas.2013.09.011

Fondo de las Naciones Unidas para la Infancia (Unicef) (2018). Política económica y social. La importancia de invertir en la niñez. Recuperado de https://www.unicef.org/spanish/socialpolicy/ index_53294.html

Fondo de las Naciones Unidas para la infancia (Unicef) (1989). Convención internacional de derechos de niños, niñas y adolescentes. Asunción, Paraguay: Unicef.

Franco, A. (2006). Tendencias y teorías en salud pública. Facultad Nacional de Salud Pública, 24(2). Recuperado de www.scielo.org.co/pdf/ rfnsp/v24n2/v24n2a12.pdf

Galand, C. y Salès-Wuillemin, E. (2009). Apports de l'étude des représentations sociales dans le domaine de la santé. Sociétés, 3(105), 3544. Recuperado de https://www.cairn.info/revue-societes-2009-3-page-35.htm

Galli, I. y Fasanelli, R. (1995). Health and Illness: A contribution to the research in the field of social representations. Papers on Social Representations, 4(1), 1-27. Recuperado de https:// www.researchgate.net/.../269689500_Health_ and_illness_a_contribution_to_the_

García-Caballero, C. (2000a). Introducción a la pediatría social. En C. García Caballero y A. González Meneses (eds.), Tratado de pediatría social (pp. 3-26). Madrid: Díaz de Santos.
García-Caballero, C. (2000b). Salud de la familia. Salud infantil. Indicadores de salud. Epidemiología de la salud de la familia: diagnóstico comunitario. En C. García Caballero y A. González Meneses (eds.), Tratado de pediatría social (pp. 44-55). Madrid: Díaz de Santos.

Gómez, R. D. (2002). La noción de salud pública: consecuencias de la polisemia. Facultad Nacional de Salud Pública, 20(1), 101-116. Recuperado de www.redalyc.org/pdf/120/12020109.pdf

González-Martínez, F., Hernández-Saravia, L. y Correa-Mulett, K. (2013). Representaciones sociales sobre higiene bucal en madres y cuidadores de hogares infantiles. Revista Cubana de Salud Pública, 39(1), 59-68. Recuperado de https:// www.scielosp.org/pdf/rcsp/v39n1/spu06113.pdf Hernández-Girón, C., Orozco-Núñez, E. y Arredondo-López, A. (2012). Modelos conceptuales y paradigmas en salud pública. Revista de Salud Pública, 14(2), 315-324. Recuperado de https://scielosp.org/pdf/rsap/v14n2/ v14n2a12.pdf DOI: https://doi.org/10.1590/ S0124-00642012000200012

Herzlich, C. (1969). Analyse d'une représentation sociale. París: Editions de l'École des Hautes Études en Sciences Sociales (EHESS).

Irwin, L. G., Siddiqi, A. y Hertzman, C. (2007). Desarrollo de la primera infancia: un potente ecualizador. Vancouver, Canadá: HELP. Recuperado de http://www.who. int/social_determinants/publications/ early_child_dev_ecdkn_es.pdf

Jiménez-Balam, D. P. (2012). Concepciones infantiles sobre el proceso salud-enfermedad en una comunidad maya de Quintana Roo. Mérida, Yucatán, México. Recuperado de http://www.mda.cinvestav.mx/ecohum/tesis_ estudiantes/TesisDJimenez12.pdf

Jodelet, D. (2013). L'apport de I'étude des représentations sociales dans le champ de la santé. 1-31. Documento inédito.

Knapp, E., León, I., Mesa, M. y Suarez, M. (2003). Representación social de la salud humana. Revista Cubana de Psicología, 20(2), 153-164. Recuperado de http://pepsic.bvsalud.org/pdf/ rcp/v20n2/07.pdf 
Laurell, A. C. (1994). Sobre la concepción biológica y social del proceso salud-enfermedad. En OPS, lo biológico y lo social: su articulación en la formación del personal de salud (pp. 1-12). Washington: OPS. Recuperado de http://www. saludcolectiva-unr.com.ar/docs/SC-043.pdf

Lima, L. y de Lemos, M. (2014). The importance of the instructions in the use of draw-and-write techniques for understanding children's health and illness concepts. Psychology, Community \& Health, 3(3), 146-157. DOI: https://doi. org/10.5964/pch.v3i3.95

López-Huerta, J. A., Álvarez-Bermúdez, J. y González-Romo, R. A. (2012). La experiencia psicosocial de padres de hijos con leucemia en un hospital público de la ciudad de San Luis Potosí. Psicooncologia, 9(1), 137-150. Recuperado de https://revistas.ucm.es/index.php/PSIC/ article/download/39143/37748

Markova, I. (1990). La presentación de las representaciones sociales: diálogo con Serge Moscovici. En J. A. Castorina (ed.), Representaciones sociales. Problemas teóricos y conocimientos infantiles (pp. 111-152). Barcelona: Gedisa.

Morin, M. (2006). Nouvelles définitions de la santé: un regard psychosocial. Spirale, 1(37), 2941. Recuperado de https://www.cairn.info/ revue-spirale-2006-1-page-29.htm

Moscovici, S. (1988). Notes towards a description of social representations. European Journal of Social Psychology, 18, 211-250. DOI: https:// doi.org/10.1002/ejsp.2420180303

Myant, K. A. y Williams, J. M. (2005). Children's concepts of health and illness: Understanding of contagious illnesses, non-contagious illnesses and injurees. Journal of Health Psychology, 10(6), 805-819. DOI: https://doi. org/10.1177/1359105305057315

Ofman, S. D. (2012). Aproximaciones al estudio de las representaciones sociales de la salud y enfermedad: el caso de la diabetes mellitus. Psicología y Psicopedagogía, 27, 34-42.

Organización Mundial de la Salud (1946). Constitución de la Organización Mundial de la Salud. Recuperado de http://apps.who.int/gb/DGNP/ pdf_files/constitucion-sp.pdf
Organización Mundial de la Salud (1986). Carta de Ottawa para la promoción de la salud. Recuperado de www.paho.org/spanish/HPP/OttawaCharterSp.pdf

Osses-Rivera, S. L., Macías Gutiérrez, C., Castaño-Galeano, S., Gómez del Castillo, D. y LópezNañez, A. (2014). Representaciones sociales que orientan prácticas de cuidado de la salud en la primera infancia: una aproximación al estado del arte. Infancias Imágenes, 13(1), 7079. Recuperado de http://revistas.udistrital.edu. co/ojs/index.php/infancias/article/view/5451

Pidgeon, V. (1985). Children's concpets of illness: Implications for health teaching. MaternalChild Nursing Journal, 14(1), 23-35.

Piko, B. F. y Bak, J. (2006). Children's perceptions of health and illness: Images and lay concepts in preadolescence. Health Education Research, 21(5), 643-653. DOI: https://doi.org/10.1093/ her/cyl034

Quevedo-Vélez, E. (2007). Henry -E. Sigerist: un pionero de la historia de la medicina y de la salud pública contemporáneas. En G. Molina Guzmán (ed.), Historia y sociología de la medicina: selecciones (pp. 13-40). Bogotá: Universidad Nacional de Colombia, Programa Interfacultad Doctorado en Salud Pública.

Rosemberg, F. (2009). La deuda latinoamericana con respecto a los niños y niñas menores de 6 años. En M. C. Torrado (ed.), Retos para las políticas públicas de primera infancia (pp. 11-28). Bogotá: Universidad Nacional de Colombia.

Rosen, G. (1993). A History of Public Health. Baltimore, MD: The Johns Hopkins University Press. DOI: https://doi.org/10.2105/AJPH.83.8.1180-a Starfield, B. (2008). Social gradients and child health. International Encyclopedia of Public Health, 6, 87-101. Recuperado de https://pdfs.semanticscholar.org/.../dcdb001b67b99552eb02f074.

Vergara, M. (2009). Representaciones sociales sobre salud de algunos grupos de jóvenes de Manizales, Colombia. Revista Latinoamericana de Ciencias Sociales, Niñez y Juventud, 7(1), 105-133. Recuperado de http://revistaumanizales.cinde.org.co/rlcsnj/index.php/ Revista-Latinoamericana/.../220 
Waitzkin, H. (2006). Un siglo y medio de olvidos y redescubrimientos: las perdurables contribuciones de Virchow a la medicina social. Medicina Social, 1(1), 5-10. Recuperado de http://www. socialmedicine.info/index.php/medicinasocial/ article/view/14/32

Wizemann, T. M. y Anderson, K. M. (2009). Focusing on Children's health. Washington: The National Academic Press.

World Bank Group (2017). Early Childhood Development: A Missed Opportunity. En W. B. Group, Sri Lanka Education Sector Assessment: Achievements, Challenges, and Policy Options (pp. 65-80). Washington: World Bank Group.
Žaloudíková, I. (2010). Children's conceptions of health, illness, death and the anatomy of the human body. School and Health, 21, 123-140. Recuperado de www.ped.muni.cz/z21/knihy/2010/26/26/.../zaloudikova_e.pdf

Бовина, И., Dvoryanchikov, N., Dany, L., Aim, M.A., Milekhin, A., Gayamova, S. y Yakushenko, A. (2018). Health representation of children and adolescents. Experimental Psychology (Russia), 11(1), 61-74. Recuperado de https://www. researchgate.net/publication/324696211_ Health_representations_of_children_and_adolescents 